\title{
Erratum to: Human Saphenous Vein Response to Trans-wall Oxygen Gradients in a Novel Ex Vivo Conditioning Platform
}

\author{
Marco Piola, ${ }^{1}$ Francesca Prandi, ${ }^{2}$ Gianfranco Beniamino Fiore, ${ }^{1}$ Marco Agrifoglio, ${ }^{3}$ \\ Gianluca Polvani, ${ }^{3}$ Maurizio Pesce, ${ }^{2}$ and Monica Soncini ${ }^{1}$ \\ ${ }^{1}$ Dipartimento di Elettronica, Informazione e Bioingegneria, Politecnico di Milano, P.zza Leonardo da Vinci 32, 20133 Milan, \\ Italy; ${ }^{2}$ Unità di Ingegneria Tissutale, Centro Cardiologico Monzino-IRCCS, Via Parea 4, 20138 Milan, Italy; and ${ }^{3}$ Dipartimento \\ di Scienze Cliniche e di Comunità, Università di Milano, Via Parea 4, 20138 Milan, Italy
}

Erratum to: Annals of Biomedical Engineering (@2015) DOI: $10.1007 /$ s10439-015-1434-0

This erratum is to correct Fig. 3. The corrected version of Fig. 3 is presented here. Figure 3a shows the results of the open-loop experimental campaign, where theoretical and measured changes in $\mathrm{O}_{2}$ concentration under various flow rate conditions are compared; while
Figs. $3 \mathrm{~b}$ and $3 \mathrm{c}$ report the results of the de-oxygenator validation in the closed loop configuration. Figure $3 \mathrm{~b}$ shows the trend of the $\mathrm{O}_{2}$ concentration within the extra-adventitial chamber as a function of the $\mathrm{O}_{2}$ saturation imposed within the de-oxygenator module. Figure $3 \mathrm{c}$ shows the results of the experiments carried out to test the system's behavior in transient conditions. (a)

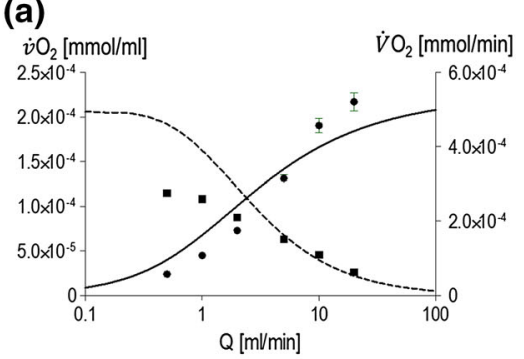

(b)

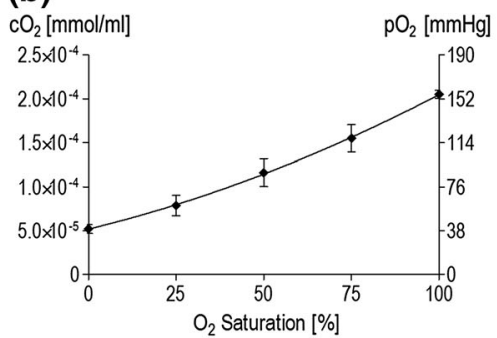

(c)

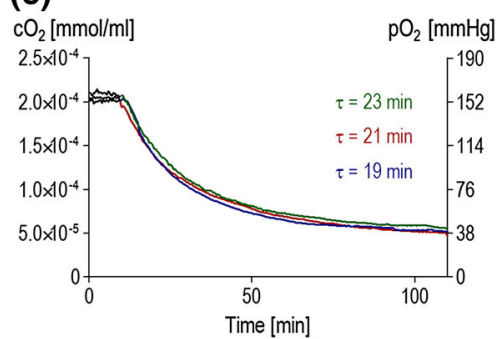

FIGURE 3. (a) Comparison between mathematical model (lines) and experimental data (squares and circles) regarding the specific mass transfer $\left(\dot{v O}_{2}\right)$ and the mass transfer rate $\left(\dot{V O}_{2}\right)$ behavior of the de-oxygenator module. Data are plotted in a semilogarithmic scale as function of the recirculating flow rate $(Q)$. (b) Trend of the oxygen concentration within the extra-adventitial chamber as a function of the $\mathrm{O}_{2}$ saturation imposed within the de-oxygenator module. (c) Results of the experiments carried out to test the system's behavior in transient conditions showed an average value of 21 min for the system's time constant, which is in fair agreement with the results obtained with the model used for the dimensioning of the de-oxygenator module. 